\title{
Formación del profesor de primaria en educación para la salud
}

\section{Primary teacher training in health education}

\author{
Esther Charro-Huerga ${ }^{(1)}$ y María Elena Charro ${ }^{(2)}$ \\ (1) Área de Didáctica de las Ciencias Experimentales. Facultad de Educación. Universidad de \\ Valladolid. Segovia. España. \\ (2) Área de Didáctica de las Ciencias Experimentales. Facultad de Educación y Trabajo Social. \\ Universidad de Valladolid. Valladolid. España
}

Resumen: La Educación para la Salud (EpS) es la materia que fomenta la vida sana en las escuelas, y que ha de ser incorporada por los docentes. Dada la importancia que tiene la salud en nuestra sociedad, se espera que los profesores estén debidamente preparados, pero desafortunadamente, hoy en día la asignatura de EpS es la mayoría de las veces una materia optativa o inexistente en los planes de estudio del Grado de Maestro en Educación Primaria en las universidades españolas. En este trabajo, tras un análisis de la situación actual en relación a la Salud y su educación, se propone que la asignatura de EpS sea considerada un pilar necesario para adquirir las competencias por los profesores en formación.

Palabras clave: Educación para la Salud; Enseñanza de las ciencias, Formación del profesorado, Grado de Maestro de Educación de Primaria.

Abstract: Health Education is the subject that promotes a healthy life in the schools, which has to be implemented by the teachers among the students. Given the importance in our society, a good training in health for the teachers is expected. Unfortunately, nowadays Health Education is either an optative subject or is not included in the Grade of Primary Teacher Education in the faculties of Education in the Spanish universities. In this work, after the analysis of the state of the art in this topic, Health Education is proposed to be considered among the main pillars needed for get the competences by the training teachers.

Keywords: Health Education, Science teaching, Teacher Training, Grade of Primary Teacher Education.

(Fecha de recepción: marzo, 2017, y de aceptación: junio, 2017)

DOI: 10.7203/DCES.32.9968 


\section{Introducción: la importancia de la educación para una sociedad sana.}

La salud es un bien social que conviene conservar y promover dado que si sus miembros no están sanos ésta se ve abocada a consumir más recursos y resulta ser menos productiva. Es, por lo tanto, clave que la sociedad vele por la salud de su población afín de poder evolucionar y desarrollarse en todos sus ámbitos. Dado el gasto económico que supone para una sociedad la sanidad, los gobiernos son conscientes cada día más de la repercusión que tiene fomentar hábitos de vida saludable entre sus ciudadanos. De este modo, a lo largo de la historia, el concepto de salud ha variado, pasando de estar centrado en la ausencia de enfermedad a referirse a una buena salud emocional y psíquica de las personas. Sin embargo, en la sociedad actual, los principales problemas de salud se deben a unos hábitos de vida poco saludables, como por ejemplo el tabaquismo. Pero si hay que destacar una lacra en nuestras sociedades desarrolladas actuales es precisamente el sobrepeso, con todas sus connotaciones y enfermedades asociadas. Así, las estadísticas indican que el $30 \%$ de la población mundial es obesa o tiene sobrepeso, que la obesidad está implicada en el $5 \%$ de los fallecimientos. El impacto económico que tiene este problema supone un gasto del 2,8\% del PIB mundial. Así, los países ricos destinan de un 2 a un $7 \%$ de su gasto en salud a tratar afecciones directamente relacio- nadas con el sobrepeso, porcentaje que se eleva hasta el $20 \%$ si se incluye el tratamiento de las enfermedades asociadas a él, como la diabetes.

Sin embargo, la obesidad no es un problema exclusivo de la fase adulta, ya que las estadísticas más recientes refieren que el problema se está extendiendo a los adolescentes y los niños. Así, varios estudios ponen de manifiesto las carencias alimentarias que tienen los niños en edad escolar: el desayuno suele ser insuficiente y poco equilibrado; escaso consumo de frutas, verduras y hortalizas, pescado o legumbres, frente al exceso en el de carne, comidas preparadas (pizzas, hamburguesas), golosinas o bollería industrial (Delgado y Lozano, 2001; Díez et al 2007; Banet y López, 2010). Es alarmante y preocupante constatar estos hechos y máxime cuando los individuos están en una fase tan crucial de crecimiento. Sin embargo, la solución a la obesidad no es ni mucho menos simple. Según un reciente estudio realizado por el "Instituto de Investigaciones Globales de la consultora McKinsey" (MGI, 2014), ningún país, en las tres últimas décadas, ha conseguido reducir su porcentaje de obesos. De nada han servido los esfuerzos para concienciar a la población y las autoridades sanitarias no se han mostrado capaces de revertir esta tendencia al sobrepeso que invade las sociedades occidentales.

Por eso, mejorar la salud de la población se ha convertido en una prioridad en estos momentos. Evidencias científicas, que no conjeturas, se pueden encontrar 
en estudios recientes, como el publicado por la revista Plos ONE (Krueger et al 2015) que certifica la relación entre el nivel educativo y la salud. El estudio concluye que el número de muertes atribuibles a un bajo nivel educativo en EEUU es comparable a las que provoca el tabaco. Así pues, según el mencionado estudio, el número de vidas que podrían haberse salvado en EEUU simplemente con una correcta educación para la salud se estima que podría haber sido de 145.243, sólo en el año 2010. Por lo tanto, a largo plazo y para mejor la salud de una sociedad de forma definitiva, la educación es el vehículo principal y más eficaz para conseguir instaurar hábitos de vida saludables. Entonces, parece obvio que es necesario fomentar los hábitos saludables de la sociedad a través de una adecuada educación en temas de salud, y así, la Educación para la Salud según la Organización Mundial de la Salud (WHO) es definida en los siguientes términos:

"La Educación para la Salud comprende las oportunidades de aprendizaje creadas conscientemente que suponen una forma de comunicación destinada a mejorar la alfabetización sanitaria, incluida la mejora del conocimiento de la población en relación con la salud y el desarrollo de habilidades personales que conduzcan a la salud individual y de la comunidad." (WHO, 1998).

Hoy en día, la Educación para la Salud (EpS) se ha convertido en un problema económico-social con numerosos actores implicados, que precisan de la colaboración y de la elaboración de un plan multidisciplinar para lograr el objetivo de mejorar la salud de la población. Por eso, actualmente se reconoce cada vez más la importancia de la EpS como instrumento imprescindible en la promoción de la salud de las poblaciones.

En este trabajo, se cuestiona si la formación del profesorado en su paso por las aulas universitarias es acorde a la labor docente que han de desempeñar en el ámbito de la educación para la salud. Para ello, en primer lugar se aborda el papel que tiene la escuela en la educación y promoción de la salud. En segundo lugar, se analiza el currículo de Educación Primaria según la actual ley LOMCE (BOE, 2013) y en relación a los aspectos relativos a la salud. Consecuentemente, en tercer lugar, se expone el estado actual de los planes de estudio del Grado de Maestro en Educación primaria en las universidades españolas afín de constatar si se contempla una formación en educación para la salud para los futuros profesores de primaria. Para finalizar, y en base a las opiniones de los docentes, recogidas en varios estudios, se reflexiona sobre la importancia de formar al profesor, la necesidad de hacerlo y del papel que se espera de él en la escuela.

\section{El papel de la escuela en la educación y promoción de la salud}

Autores como Gunay et al (2015) señalan que los temas sobre salud es 
importante abordarlos desde edades tempranas, tanto dentro de la familia como desde el colegio. De esta forma, la escuela constituye uno de los principales escenarios educativos para desarrollar actividades saludables (Leger, 2001, Gutiérrez, 2003, Perea Quesada, 2001), donde se trabaja con alumnos que están en período de formación física, psíquica y social, y que poseen una gran capacidad para el aprendizaje y la asimilación de hábitos, y que por ende está abierta a la familia y a la comunidad. Por lo tanto, dada la importancia que tiene la escuela de cara a mejorar la salud en la sociedad, como señalan diversos autores también a nivel internacional (Wyrick et al. 2001; Jourdan et al 2008; Seffrin, 2008, Inel et al 2011), es necesario implementar en la escuela un programa normalizado que permita a todos los ciudadanos de un país adquirir conocimientos, actitudes, habilidades y destrezas suficientes para enfrentarse a la solución de los problemas de salud de su vida cotidiana (García et al 2000; Castillo et al 2003, Escámez Sánchez 2001). No hay que olvidar, que la EpS es de interés a distintos niveles educativos, desde la educación infantil hasta la secundaria como demuestran diversos estudios (Aramendi et al 2014, Vega Fuente et al 2015). Así, la EpS deberá estar encaminada a promover estilos de vida saludable y es una tarea que los profesores deberán integrar en el conjunto educativo, siendo los centros de enseñanza a través de sus profesores los que desempeñen un papel importante en la configuración de los distin- tos hábitos saludables y de los valores y conductas sociales deseables en una sociedad del siglo XXI.

En un principio, el papel de la institución educativa se centró en acciones preventivas, siendo la modificación de conductas individuales su principal objetivo (drogas, alcohol, tabaco, alimentación, actividad física y salud mental). En 1990, la Organización Mundial para la Salud (WHO) propuso una serie de criterios generalistas para anexar la promoción de la salud al currículo escolar (Richonnier, 1990). El objetivo perseguido consistía en mostrar a la comunidad educativa modelos de vida saludables así como contextos favorables a la salud. Con el trascurrir del tiempo, las líneas generales se han ido concretando en líneas de acción específicas como, por ejemplo, el desarrollo de buenas relaciones interpersonales en la escuela, la mejora de la salud y el bienestar del personal del centro, la promoción de la autoestima y autonomía de alumnado, el cuidado de los aspectos físicos y organizativos del centro, la consideración del personal de la escuela como modelo en temas de salud e incluso la rehabilitación fisioterapéutica (Calvo y Gómez, 2013). La puesta en práctica de estas conductas saludables en los centros escolares viene a constituir el currículum oculto del centro y comprende el conjunto de valores, normas y actitudes que de manera implícita, están presentes y conforman el microclima del mismo.

En el año 2008, el Ministerio de Sanidad español presentó un estudio 
(Salvador Llivinia, 2008) en el que se identifican los avances conseguidos en las distintas comunidades autónomas a lo largo de 20 años (desde 1986 hasta 2006) en materia de Educación y Promoción para la Salud en la escuela en España, así como las necesidades y retos encontrados en el 2008. En dicho estudio se indica que durante los años 90 y primera década del 2000 se crearon plataformas operativas para abordar un tema tan importante como la EpS en las escuelas, junto con planes de formación para el profesorado en activo y se definieron los temas identificados en aquel entonces como prioritarios: trastornos de la alimentación, educación sobre drogas y sexualidad, y actividad física, y también se crearon, algunas guías de trabajo para intentar fomentar proyectos en este ámbito. Sin embargo, se identificaron obstáculos importantes tales como que la EpS no es una prioridad educativa en las políticas educativas, pese al reconocimiento de su importancia y como consecuencia se deja a la "voluntariedad" de las diversas instituciones y de los educadores, el fomento de la misma. El profesorado no se ve como agente sanitario, ya que no dispone de formación para ello, y delega todo lo relacionado con salud en el personal sanitario. La EpS compite con un conglomerado de contenidos transversales que saturan y hacen complicada su práctica en nuestras escuelas. Por último, la apretada agenda de los profesionales educativos y la falta de implicación familiar hacen que la EpS no tenga el peso que le corresponde en la educación de los escolares.

Ese mismo año se publica el trabajo de Davó et al (2008) que recoge una revisión de los estudios publicados entre los años 1995 y 2005 acerca de la promoción y educación para la salud en las etapas de infantil y primaria de la escuela española. Este estudio permite conocer las características de las intervenciones realizadas, donde se encontró que los programas de EpS tienen una perspectiva más preventiva de la enfermedad que de la promoción de la salud, no siendo frecuentes en las etapas de infantil y primaria.

Años más tarde, se publica otro estudio (Jiménez-Torres et al. 2013) donde se ha llevado a cabo una recopilación y posterior análisis de las publicaciones sobre la implementación de la EpS en las instituciones educativas españolas durante 20 años, concretamente en el período comprendido entre los años 1993 y 2013. En este trabajo, JiménezTorres et al. (2013) a partir de un total de 245 artículos, encuentran que los temas tratados corresponden a:

- Cuestiones teóricas de EpS y a encuestas sobre salud física percibida (más del 40\%).

- Promoción de la salud referidos a la actividad física y la alimentación saludable (12\%).

- Drogodependencias (9\%).

- Validación de instrumentos para medir varios aspectos relativos a la EpS (casi un 6\%). 
- Educación afectivo-sexual (casi un $5 \%$ ).

- Prevención de riesgos, $(2 \%)$.

Estos autores (Jiménez-Torres et al., 2013) también encuentran que todas estas investigaciones son promovidas en más del $50 \%$ por las universidades, en un $24 \%$ por los Centros de Salud y Hospitales, en un $10 \%$ por otras administraciones y fundaciones públicas, $\mathrm{y}$ sólo el $9 \%$ se deben a la iniciativa de las instituciones educativas no universitarias. De este modo, el trabajo de Jiménez-Torres et al. (2013) confirma que el tipo de intervención que se viene realizando en los centros educativos se orienta más a la prevención que en la promoción de la salud, y que las instituciones universitarias tienen más protagonismo que las no universitarias en la difusión de la promoción de la salud.

En base a lo anteriormente expuesto, surgen diferentes iniciativas en estos últimos años. Por ejemplo, las empresas privadas de seguros, a quiénes le repercute de forma económica muy relevante, son conscientes de la importancia de la EpS en la escuela, y editan cuadernos y guías docentes de actividades gratuitas para su uso escolar, como por ejemplo Mapfre (Fundación Mapfre, 2014). También los diferentes gobiernos autonómicos, además de las guías didácticas y de consejos que se editan de forma física y on-line, llevan de forma periódica campañas para "atacar" de forma concreta determinados aspectos de la EpS. Este tipo de campañas tienen un valor importante a la hora de reforzar determinados aspectos de la EpS, pero presentan limitaciones, como la voluntariedad de adhesión por parte de los diferentes centros educativos a los que se oferta y el enfoque a un determinado aspecto concreto aislado dentro de la gran amplitud de la EpS. Esto refuerza la tendencia de la que se hablaba en el informe ministerial de 2008 (Salvador Llivinia, 2008) en el que se facilitan recursos por parte institucional, pública y privada, pero sigue dejándose a la iniciativa por parte de los profesionales de la educación llevarlo a la práctica curricular.

\section{La Educación para la Salud en el currículo de Educación Primaria}

La EpS ha estado siempre presente en la escuela y normalmente asociada el área de las Ciencias de la Naturaleza. La higiene, la sexualidad, la prevención de enfermedades, los efectos del alcohol y los narcóticos, eran, entre otros, temas aptos para ser incluidos en los cursos de biología según Linville (1989). De modo que conceptos de salud como vacunaciones, infecciones, inmunidad, alimentación,..., se vienen tratando en el aula, o bien porque estaba contemplado en los programas oficiales, o por el interés del profesorado, que veía en ellos su potencial educativo y la necesidad social de incluirlos en el conocimiento escolar (Gavidia, 2001). Así, según la hasta hace poco legislación española en materia de educación LOGSE (BOE 2007), el área de Conocimiento del Medio Natu- 
ral, Social y Cultural era la encargada de integrar los contenidos de salud y desarrollo personal, en lo que constituía el Bloque 3, en cualquiera de los tres ciclos de la Educación Primaria, y que se definía, de manera global, como "Conocer y apreciar el propio cuerpo y contribuir a su desarrollo, practicando ejercicio físico y los hábitos elementales de higiene y alimentación, valorando positivamente su repercusión sobre la salud y la calidad de vida”.

Pero, en la actual ley LOMCE (BOE, 2013), los conceptos asociados a la EpS desaparecen de los bloques de contenido específicos de las asignaturas y se enmarcan dentro de los contenidos transversales. Pero, por otra parte, en la nueva ley existen algunos contenidos puntuales de EpS que han sido incluidos en determinadas asignaturas como en la Educación Física, y que son: el deporte, fitness y wellness. En cualquier caso, es dentro de los contenidos transversales que deben fomentar las administraciones educativas donde se incluyen concretamente los siguientes conceptos relacionados con la EpS: desarrollo sostenible y medio ambiente; actividad física y dieta equilibrada; vida activa, saludable y autónoma; educación y seguridad vial; y el abuso sexual, el cuál es específicamente mencionado como concepto a tratar. Así, la transversalidad, que plantea la distribución de la programación de la EpS por todas las áreas del currículo, aparece por la demanda de la sociedad de incorporar nuevos temas relacionados con la salud en el currículo escolar y sobre todo para evitar quitar tiempo a las áreas ya establecidas, y es por tanto una responsabilidad que debe asumir toda la comunidad educativa (Busquets et al 1993). Sin embargo, el concepto de transversalidad ha cambiado en poco tiempo, gracias a las aportaciones realizadas por el profesorado y la experiencia adquirida al generalizar su tratamiento (Gavidia, 2001). En un principio, se contemplaban ciertos contenidos de EpS distribuidos en diversas disciplinas. De esta forma, las materias transversales no pretenden desmontar las asignaturas existentes tradicionalmente, sino hacer que sus límites estén más diluidos a fin de acercarnos a una realidad compleja, la cual es abordada con una intencionalidad globalizadora (Yus, 1997). Ahora, estos contenidos, considerados transversales, han pasado a representar el conjunto de valores, actitudes y comportamientos que deben ser educados, lo que puede ser considerado como símbolo de innovación y de apertura de la escuela a la sociedad (Gavidia, 1996).

Por otro lado, llama la atención que se indique que los temas relacionados con la EpS deben ser asumidos en su diseño, coordinación y supervisión por el profesorado con cualificación o especialización adecuada en dichos ámbitos. En su momento, para desarrollar los aspectos transversales relacionados con la EpS fue de especial relevancia contar con el Manual de Formación del Profesorado (Metcalfe et al 1995), que propone una metodología formativa basada en la participación y aporta el material necesario para el desarrollo 
de cada tema (Alonso-Blanco y Villalvilla-Soria, 2007). Pero sin duda, el libro de texto es el recurso sobre el que se apoya el maestro a la hora de impartir los contenidos de salud que se señalan en la ley educativa del momento. Cabe entonces preguntarse, si los textos escolares vinculados a la enseñanza de las ciencias naturales recogen los contenidos que serían deseables para una adecuada educación para la salud escolar y si realmente sirven de apoyo a los docentes. Existen estudios que analizan cómo se contempla la EpS en los textos de enseñanza primaria, como por ejemplo el de Gavidia (2003), donde tras un análisis exhaustivo de muchos libros, se concluye que los actuales textos no son un referente adecuado para que el profesorado desarrolle la EpS en el aula. Se indica, por ejemplo, que los temas más tratados son los de higiene, y la metodologçia no contempla contenidos procedimentales ni criterios actitudinales. Además, concluye Gavidia (2003) estos manuales escolares no fomentan actitudes y conductas. Similares conclusiones encuentran los autores Barrio et al (2008) tras analizar 100 libros de texto y varios cientos de mensajes sobre salud. En estos recursos, se trata fundamentalmente la alimentación y el ejercicio físico, pero muy poco sobre obesidad infantil.

\section{La Educación para la Salud en la formación de los maestros de primaria}

En el contexto europeo, desde la firma de la Declaración de Bolonia en junio de 1999 y, posteriormente, con la Declaración de Berlín en 2003, se implanta el Espacio Europeo de Educación Superior (EEES) en nuestras universidades. Este hecho ha supuesto una magnífica oportunidad que permite a las universidades adaptarse a la nueva realidad social, la denominada Sociedad del Conocimiento o Sociedad del Saber (Sanz, 2012), y ha conducido a reformas en el ámbito universitario en diversos países. De este modo, en la etapa pre-Bolonia, la formación del maestro en España se había caracterizado por estar centrada en la enseñanza más que en el aprendizaje. Con la implantación del EEES, las universidades orientan su formación hacia el aprendizaje de competencias y esto conlleva un nuevo modelo de formación. De este modo, se crea un nuevo paradigma educativo que tiene como punto focal a la visión competencial. El nuevo modelo otorga una gran responsabilidad a las universidades para que formulen criterios académicos, competenciales y de cualificación profesional que respondan a la demanda del mercado laboral europeo y que permitan responder a los retos educativos, laborales y sociales actuales (García Manjón y Pérez López, 2008).

En la actualidad, en España, el Título de Grado de Maestro en Educación Primaria es el único que capacita para impartir clases a los alumnos de Educación Primaria. Los maestros durante cuatro años, han de adquirir formación 
teórica y práctica acordes con los objetivos previstos en las leyes que regulan sus funciones y han de estar capacitados para participar activamente tanto en el diseño como en el desarrollo de proyectos de innovación que contribuyan a la permanente mejora cualitativa y cuantitativa del Sistema Educativo. Según el Libro Blanco (ANECA, 2004) en el Grado de Maestro de Educación Primaria las competencias que ha de adquirir el futuro profesor y que están relacionadas con la salud pública están referidas a las actividades de educación y promoción para la salud. Así, con la llegada del EEES se esperaba que la asignatura de EpS, tradicionalmente optativa, fuera obligatoria en los nuevos planes de estudio en la formación de maestros en Educación Primaria, como apunta Cubero et al (2011), pero no ha sido de este modo. Una revisión de las asignaturas ofertadas en los planes de estudio de las Titulaciones de Grado de Maestro en Educación Primaria en las principales universidades españolas ha permitido elaborar la Tabla I. En esta Tabla se muestra una relación de universidades españolas públicas y la inclusión o no de la Educación para la salud (u otra similar) en el Grado de Maestro de Educación Primaria.

\section{Tabla I. Asignaturas relativas a la EpS en el Grado de Educación Primaria en varias universidades públicas españolas.}

(Fuente: Elaboración propia)

$\begin{array}{llcc}\text { Universidad } & \text { Asignatura } & \text { Carácter } & \text { ECTS } \\ \text { Alcalá* } & \text { Condición física y salud en el aula de educación física } & \text { Optativa } & 6 \\ \text { Alicante } & \text { No consta } & - & - \\ \text { Almería } & \text { Actividad física, hábitos saludables y calidad de vida } & \text { Optativa } & 6 \\ \text { Autónoma de Madrid* } & \text { La condición física y la salud en la edad escolar } & \text { Optativa } & 6 \\ \text { Barcelona } & \text { No consta } & - & - \\ \text { Burgos } & \text { Educación para la Salud } & \text { Optativa } & 5 \\ \text { Cádiz } & \text { Actividad física y salud } & \text { Optativa } & 6 \\ \text { Cantabria } & \text { No consta } & - & - \\ \text { Castilla-La Mancha } & \text { Condición física y salud } & \text { Optativa } & 6 \\ \text { Complutense de Madrid } & \text { No consta } & - & - \\ \text { Córdoba } & \text { Educación Física y salud } & \text { Optativa } \\ \text { Extremadura } & \text { Motricidad, actividad física y salud } & \text { Optativa } \\ \text { Granada } & \text { No consta } & - & - \\ \text { Huelva } & \text { Actividad física y salud } & \text { Optativa } & 6 \\ \text { Illes Balears } & \text { Educación física y hábitos de vida saludable } & \text { Obligatoria } \\ \text { Jaén } & \text { Actividad física y salud } & 6 \\ \text { Las Palmas de Gran } & \text { Didáctica de las ciencias naturales y de la educación para la } & \text { Optativa } & 6 \\ \text { Canaria } & \text { salud, biodiversidad y entorno } & \text { Obligatoria } \\ \text { La Coruña } & \text { Didáctica de la Educación para la Salud } & 6 \\ \text { La Rioja } & \text { Actividad física y salud } & \text { Optativa } & 4,5 \\ & & \text { Optativa } & 4,5\end{array}$




\begin{tabular}{llcc} 
León & No consta & - & - \\
Lleida & Salud y malestar docente & Optativa & 6 \\
Málaga & Actividad física y salud & Optativa & 6 \\
Murcia & Educación física y salud & Obligatoria* & 3 \\
Navarra & Educación para la salud & Obligatoria & 6 \\
País Vasco & No consta & - & - \\
Oviedo & Primeros auxilios, salud y prevención de accidentes en la escuela & Optativa & 6 \\
Salamanca & Higiene y primeros auxilios de la actividad física y su didáctica & Optativa & 6 \\
Santiago de Compostela & No consta & - & - \\
Sevilla & No consta & - & - \\
Valencia & Didáctica de la educación física para la salud & Optativa & 6 \\
Valladolid & Educación para la Salud & Optativa & 6 \\
Zaragoza & No consta & - & - \\
\hline
\end{tabular}

*Sólo en la mención de Educación Física

El número de universidades españolas es en estos momentos de unas 80 , siendo 50 de ellas públicas y las restantes privadas. Por otro lado, todas y cada una de las universidades públicas españolas ofertan el Grado de Maestro en Educación Primaria. Además, recientemente, algunas universidades españolas ofertan también dobles grados de Maestro en Educación Primaria e Infantil. A su vez, la mayoría de las universidades públicas españolas disponen de diferentes campus, donde es frecuente encontrar también en cada uno de ellos la impartición del Grado de Maestro en Educación Primaria. Se dispone, así, de un amplio abanico de centros que ofertan esta titulación, habitualmente Facultades de Educación o de Ciencias de la Educación, y que se distribuye por todo el territorio español. De este modo, en la Tabla 1, donde se recoge un $64 \%$ de las universidades públicas españolas, se observa, que en la actualidad, la presencia de la asignatura de EpS, u otra similar, se contempla mayoritariamente como optativa.
Sólo se observan algunas excepciones, como puede ser en las universidades de las Palmas de Gran Canaria y Navarra donde es obligatoria, y también en las que la salud se aborda dentro de la mención de Educación física, dentro de la cual, la asignatura puede a su vez ser optativa o no. En algunos casos, incluso, según la Tabla I, es inexistente. Por otro lado, sin embargo, los contenidos relativos a la EpS en asignaturas con diferente denominación sí son contemplados en el grado de Educación Infantil, como puede verse en la Tabla II, donde por supuesto forma parte de la formación básica del futuro profesor de Educación Infantil.

\section{Discusión: la importancia de formar al docente en Educación para la Salud}

La importancia de llevar a cabo una formación de los docentes en materia de salud es un tema ya abordado hace varios años pero no por eso ha dejado de ser de reciente actualidad como lo 


\section{Tabla II. Asignaturas relativas a la EpS en el Grado de Maestro de Educación Infantil en varias universidades españolas.}

(Fuente: Elaboración propia)

\begin{tabular}{llcc} 
Universidad & Asignatura & Carácter & ECTS \\
A Coruña & Enseñanza del cuerpo humano y de hábitos de vida saludables & Optativa & 4,5 \\
Alicante & No consta & - & - \\
Autónoma de Madrid & No consta & - & - \\
Barcelona & Infancia, salud y alimentación & FB(a) & 6 \\
Burgos & Salud y alimentación infantil & FB & 6 \\
Cantabria & Promoción del desarrollo infantil saludable & Obligatoria & 6 \\
Complutense de Madrid & Fundamentos y didáctica de la alimentación & FB & 6 \\
Extremadura & Educación para la Salud & optativa & 6 \\
Granada & No consta & - & - \\
La Rioja & Infancia, salud y alimentación & FB & 9 \\
León & Nutrición y desarrollo & FB & 6 \\
Málaga & Salud, higiene y alimentación infantil & FB & 6 \\
Murcia & No consta & - & - \\
Oviedo & Desarrollo, comportamiento y salud & FB & 6 \\
Salamanca & No consta & - & - \\
Santiago & Infancia salud y alimentación & FB & 6 \\
Sevilla & Psicopatología del desarrollo y salud infantil & FB & 6 \\
Valencia & Biología del desarrollo & FB & 4 \\
Valladolid & Infancia, salud y alimentación & FB & 6 \\
\hline
\end{tabular}

(a) Formación básica

demuestran trabajos como los de DavóBlanes et al (2016), Gunay et al. (2015), Paakkari et al. (2015), o (Rodrigo et al 2013) Así, por ejemplo, a nivel institucional, en algunas universidades españolas como la Complutense de Madrid se ofertan asignaturas para la formación del maestro enfocadas a la educación alimentaria a raíz de los altos índices de obesidad que se están dando entre la población.

Haciendo un poco de historia, ya hace dos décadas, Rodrigo (1995) realizó un estudio para conocer las concepciones de los futuros profesores de primaria sobre la EpS, donde la gran mayoría de los entrevistados manifestaban no tener formación previa en esa temática, y creían que era necesaria la participación de sanitarios en este proceso del que mayoritariamente opinaban había de ser de índole teórico-práctico. Pero la necesidad de incluir e impartir esta asignatura en los planes de estudio de los futuros maestros no es exclusivo de nuestro país. Así, Thackeray et al. (2002) señalan en su trabajo que la mayoría de los profesores que tomaron parte en su 
estudio dijeron que en su formación no recibieron ninguna preparación sobre contenidos de salud o métodos de enseñanza de la misma. Esto es usual en los programas universitarios de educación primaria, donde hay poca o ninguna formación en contenidos de salud y su metodología, como ha sido expuesto anteriormente y también por otros autores (Anspaugh y Ezell, 2001). También Cubero et al (2011) encontraron en su estudio con estudiantes universitarios de magisterio, que un alto porcentaje de ellos poseía escasos conocimientos básicos en EpS, pero esto contrastaba, a su vez, con una incipiente demanda e interés por la enseñanza de los contenidos sobre salud. Así mismo, trabajos recientes como los de Alonso-Peña y Campo (2014) también analizan la necesidad de formación en temas de salud de los profesores de Educación Primaria para elaborar un programa formativo dirigido a este colectivo y refieren la necesidad que tiene el profesorado de primaria en materia de salud y en particular, primeros auxilios. Más recientemente, y como prueba de la aún demandada necesaria formación en la educación para la salud a nivel escolar, y también, la necesaria colaboración entre los distintos agentes implicados en la misma, encontramos el estudio de Davó-Blanes et al (2016). En este estudio, los autores llevan a cabo un análisis exploratorio con un grupo de profesores de primaria y en relación a las actividades de salud realizadas en la escuela, donde se concluye, en opinión de los docentes, que "incrementar su formación en salud, implicar a los padres y madres, profesionales sanitarios y la administración educativa fomentaría la educación para la salud en la escuela".

Por otro lado, lo habitual es que los programas de EpS escolar necesiten la colaboración entre el personal sanitario y el docente, lo que requiere que se compartan conceptos e ideas. Hace años, Gavidia et al. (1993) señalaban que se debe contar con la participación de padres, sanitarios y demás agentes asociados para una Educación para la salud efectiva. También, diversos organismos e instituciones del ámbito de la salud reconocen que los maestros tienen competencias en salud pública que van más allá de las relacionadas con la tradicional educación sanitaria (Cubero et al 2011). Además, otros estudios como el de Talavera y Gavidia (2013) ponen de manifiesto la opinión que realmente tienen los agentes implicados en la EpS, y es tan simple como que "los docentes opinan que la familia y el personal sanitario deben tener mayores responsabilidades que ellos mismos, y por el contrario, los sanitarios opinan que la responsabilidad principal debe recaer entre el profesorado".

Por otro lado, autores como Gavidia (1993) y Oliva (2011) señalan que es necesario que el profesorado cuente, entre otras cosas, con conocimientos sobre los temas básicos de salud, competencias para desarrollar estos conocimientos, habilidades que puedan ser aprendidas, y relaciones con las agencias locales y las organizaciones de la comunidad que participen en el contexto socio-educativo de los estudian- 
tes. Pero, tanto en la etapa pre-Bolonia como en la actualidad, la formación en los temas de salud no se contempla de forma obligatoria en los planes para la formación inicial del profesorado, y la que poseen los profesores ha sido adquirida de manera "ambiental" e intuitiva (Gavidia, 1998; Aldrete et al 2002; Talavera y Gavidia 2013). Según señalaba Gil (1991), a las competencias que deben poseer los docentes para su desarrollo profesional hay que sumarles las específicas de la EpS, que según indican Gutiérrez (2003) deberían ser fortalecidas por los programas de formación de las instituciones de enseñanza. En este sentido, Davó et al (2011) realizan un análisis de las competencias y contenidos básicos de salud pública en los programas de magisterio de diversas universidades, del que concluyen que existe una necesidad de introducir propuestas de mejora. Por lo tanto, la formación de los profesionales docentes, tanto inicial como continuada, se constituye como un pilar básico para la adopción de las nuevas estrategias y metodologías que favorezcan la promoción de la salud en la escuela, la cual es el mejor ámbito para instaurar un entorno físico y psicosocial adecuado en el que el alumnado aprenda y viva comportamientos críticos, solidarios y saludables (Talavera y Gavidia 2007).

\section{Conclusiones}

Este estudio releva una realidad, y es que, sin lugar a dudas, el maestro es el agente fundamental de la EpS en la escuela, pues a partir de la entrada de los niños en la misma la responsabilidad de la educación del alumno pasa a ser compartida entre familia y maestros (Castillo et al 2003). La importancia de promover la EpS en la escuela, y por lo tanto, en los diversos niveles educativos de la enseñanza obligatoria, desde las edades más tempranas, y de manera continua e ininterrumpida, queda sobradamente justificada. Para lograr sus inherentes objetivos educativos hace falta que los estudiantes y profesores sean saludables física, mental y socialmente, ya que de esta forma, se garantizará a largo plazo una sociedad sana, cuando estos individuos sean adultos.

A lo largo de este estudio ha quedado patente la necesidad de una formación inicial del profesor de Educación Primaria en EpS. Esta formación ha de contribuir inexorablemente a que el docente adquiera unas competencias en materia de salud que han de gestarse a partir de sus experiencias como estudiante, en su paso por las aulas universitarias. Las competencias mencionadas podrán, difícilmente, adquirirse sólo en base a una experiencia profesional sin formación previa. Los conocimientos con los que cuentan los estudiantes de Grado de Maestro en Educación Primaria "a priori" son fundamentalmente los relativos al cuerpo humano, su funcionamiento y su relación con el medio. Estos conocimientos son necesarios, pero existe una serie de carencias educativas en relación a la salud que son mejorables a través de una formación adecuada del profesorado. Este hecho, 
por lo tanto, requiere que los docentes tengan además de los conocimientos en EpS necesarios, la habilidad e interés para implementar estrategias educativas en el campo de la salud (Talavera y Gavidia, 2007). Por eso, la EpS en la formación del maestro es una disciplina ideada para que los futuros educadores sean capaces de programar, desarrollar y evaluar un plan de trabajo en el aula y en el área de la salud para Educación Primaria, lo cual es sin duda, el otro factor importante en relación con la implementación de estrategias educativas en el ámbito de la salud.

De este modo, la presencia de la EpS en los planes de estudio de las universidades en los Grados de Maestro en Educación Primaria parece más que justificada. Sin embargo, como se ha podido constatar, la presencia de la asignatura de EpS u otra similar en el Grado mencionado es inexistente o se encuentra como optativa en la mayoría de las universidades españolas. Hay que dejar constancia, por lo tanto, de que la inclusión de la EpS en los planes de estudio de formación de maestro de Educación primaria como optativa permite completar la formación de algunos de los futuros profesores, pero no garantiza la adquisición de estos conocimientos y competencias para el resto del alumnado que no la elige entre sus optativas. Aun así, la incorporación de la EpS como materia optativa en los planes de estudio de las universidades españolas es habitual y deseable como apunta García-Barros (2008), y sin duda contribuye a la formación docen- te. Así, desde este trabajo, y de acuerdo con otros autores (Valverde et al 2009), se propone que la EpS no se enmarque en una mera asignatura optativa, sino que adquiera un grado de importancia tal que todo futuro docente se forme en ella (Benavides et al 2006). También se quiere apostar desde este estudio, y como otros autores señalan (Molina 2006), por una coordinación adecuada entre la administración y las universidades, a fin de sistematizar la formación universitaria y poder contar con profesionales adecuadamente preparados y cualificados en EpS.

\section{Referencias bibliográficas}

ALDRETE,M.G.;VALADEZ,I.;CABRERA, C.; MENDOZA, P.; PANDO, M.; ARANDA, C. (2002). La Educación para la Salud en las Escuelas de Educación Básica. Investigación en Salud, artículo de investigación $\mathrm{n}^{\circ} 6$. ALONSO-BLANCO, C.; VILLALVILLA SORIA, D.J. (2007). El reto de la Educación para la Salud en la comunidad escolar. Multiárea, 2, 25-63.

ALONSO-PEÑA, N.; CAMPO BRIZ, M. (2014). Necesidad percibida de formación sanitaria de los profesores de Educación Primaria. Nuberos Científica. 2(13), 25-31.

ANECA (2004). Libro Blanco. Título de Grado en Magisterio, vol. I y II. Madrid.

ANSPAUGH, D.; EZELL, G. (2001). Teaching Today's Health. Ed. Allyn \& Bacon, Boston, MA. 
ARAMENDI, P.; BUJÁN K.; ARBURNA, R. (2014). Educación para la Salud e invención educativa en la ESO. La percepción del alumnado. Revista española de Pedagogía. 259.

BANET, E.; LÓPEZ, C. (2010). ¿Cómo mejorar el desayuno de los escolares de educación primaria? Investigación en la Escuela, 71, 63-83.

BARRIO ET AL. (2008). Presencia de las actuales prioridades de salud infantil y adolescente en los libros de texto escolares. Gaceta sanitaria. 22 (3), 227-231. DOI: 10.1157/13123968.

BENAVIDES, F., BOLÚMAR, F., GÓMEZ-LÓPEZ, L. (2006) El Espacio Europeo de Educación Superior, una gran oportunidad para la salud pública. Gaceta sanitaria. 20, 2, 15-18. DOI: $10.1157 / 13087317$.

BOE (2007). Orden ECI/2211/2007, Núm. 173. de 12 de julio, por la que se establece el currículo y se regula la ordenación de la Educación primaria.

BOE (2013). Ley Orgánica 8/2013, de 9 de diciembre para la mejora de la calidad educativa (LOMCE). Núm. 295, de 10 de diciembre de 2013, 97858 -97921.

BUSQUETS, M.; CAINZOS, M.; FERNÁNDEZ, T.; LEAL, A.; MORENO, M.; SASTRE, G. (1993). Los temas transversales. Claves de la formación integral. Madrid: Aula XXI-Santillana.

CALVO, I.; GÓMEZ, A. (2013). El fisioterapeuta y la Educación para la Salud en la escuela. Cuestiones de Fisioterapia. Revista Universitaria de Información e Investigación en Fisioterapia, 42, 1-2.

CASTILLO, M. D.; NARANJO, B.; GIL, M. T. (2003). Implicación del profesorado en Educación para la Salud en los centros docentes. Revista de la SEMG 52, 181-189.

CUBERO, J.; CALDERÓN, M.; COSTILLO, E.; RUIZ, C. (2011). La educación para la Salud en el Espacio europeo de educación Superior. Publicaciones, 41, 51-63.

DAVÓ-BLANES, M.C., GARCÍA DE LA HERA, M., LA PARRA, D., (2016). Educación para la salud en la escuela primaria: opinión del profesorado de la ciudad de Alicante. Gaceta Sanitaria, 30(1), 31-36. DOI: 10.1016/j. gaceta.2015.07.008.

DAVÓ, M.C., ET AL (2011). Competencias y contenidos comunes de salud pública en los programas universitarios de grado. Gaceta Sanitaria, 25(6), 525-534. DOI: 10.1016/j.gaceta.2011.06.004.

DAVÓ, M.C., GIL-GONZÁLEZ, D., VIVES-CASES, C., ÁLVAREZ-DARDET, C., LA PARRA, D., (2008). Las investigaciones sobre promoción y educación para la salud en las etapas de infantil y primaria de la escuela española. Una revisión de los estudios publicados entre 1995 y 2005 . Gaceta Sanitaria, 22(1), 58-64. DOI: 10.1157/13115112.

DELGADO, A.; LOZANO, M.J. (2001). Alimentación del niño y adolescente. Guías alimentarias para la población 
española, 365-372. Madrid: Sociedad Española Nutrición Alimentaria (SENC).

DÍEZ, L.; GALÁN, I.; LEÓN, C.M.; GANDARILLAS, A.; ZORRILLA, B., ALCARAZ, F. (2007). Ingesta de alimentos, energía y nutrientes en la población de 5 a 12 años de la comunidad de Madrid: resultados de la encuesta de nutrición infantil 2001-2002. Revista Española de Salud Pública, 81(5), 543-558. DOI: 10.1590/s1135-57272007000500010.

ESCÁMEZ SÁNCHEZ J. (2001). Valores, actitudes y habilidades en la educación para la salud. Educación $X X 1,4,41-59$. DOI: $10.5944 / \mathrm{edu}-$ cxx1.4.0.363.

FUNDACIÓN MAPFRE (2014). Cuadernos. www.fundaciónmapfre.org.

GARCÍA, A.; SÁEZ, J.; ESCARBAJAL, A. (2000). Educación para la Salud, la apuesta por la calidad de vida. Madrid: Arán Ediciones S.A.

GARCÍA-BARROS, S. (2008, 9-12 de septiembre). "La formación del profesorado de Educación Infantil". Ponencia presentada en los XXIII Encuentros de Didáctica de las Ciencias Experimentales, Almería.

GAVIDIA CATALÁN, V. (1993). Consideraciones sobre la formación inicial del profesorado en educación para la salud. Didáctica de las ciencias experimentales y sociales, 7, 59-70.

GAVIDIA CATALÁN, V. (1996). «La construcción del concepto de trans- versalidad». Aula de Innovación Educativa, 55, 71-77.

GAVIDIA CATALÁN, V. (1998). Salud, Educación y Calidad de Vida. De cómo las concepciones del profesorado inciden en la salud. Ed. Magisterio: Santa. Fé de Bogotá

GAVIDIA CATALÁN, V. (2001). La transversalidad y la escuela promotora de salud. Revista Española de Salud Pública, 77, 275-285. DOI: 10.1590/s1135-57272001000600003.

GAVIDIA CATALÁN, V. (2003). La educación para la salud en los manuales escolares españoles. Revista Española de Salud Pública, 71,505-516.DOI: 10.1590/s1135-57272003000200010.

GAVIDIA CATALÁN, V., RODES SALA, M.J., CARRATALÁ BEGUER, A. (1993). La educación para la salud: una propuesta fundamentada desde el campo de la docencia. Enseñanza de las ciencias, 11(3), 289-296.

GIL, D. (1991). ¿Qué han de saber y saber hacer los profesores de ciencias? Enseñanza de las Ciencias, 9(1), 69-77.

GUNAY, Y.; CAVAS, B.; HAMURCU, H. (2015). Pre-service Teachers' Views on the Environmental Education, Human Brain and Genetics, Health and Sexual Education. Procedia - Social and Behavioral Sciences 167, 141 - 151. DOI: 10.1016/j.sbspro.2014.12.655.

GUTIÉRREZ, J. J. Coord. (2003). Formación en Promoción y Educación para la Salud. Ministerio de Sanidad y 
Consumo. Consejo Interterritorial del Sistema Nacional de Salud: Madrid. INEL, D.; GÜNAY, Y.; EVREKLI, E.; HAMURCU, H. (2011). Pre-service Teachers' Opinions about the Effects of Harmful Habits, Drug Use and Physical Activities on Human Health. Western Anatolia Journal of Educational Science, 349-358.

JIMÉNEZ-TORRES, M. G.; GUERRERO-RAMOS, D.; LÓPEZ-SÁNCHEZ, M. (2013). Publicaciones sobre Educación para la Salud en las instituciones educativas españolas (19932013) Revista Iberoamericana de Psicología y Salud, 4.

JOURDAN, D.; SAMDAL, O.; DIAGNE, F.; CARVALHO, G. (2008). The Future of Health Promotion in Schools Goes Through The Strengthening of Teacher Training at a Global Level. Promotion and Education 15(3): 36-38. DOI: $10.1177 / 1025382308095657$.

KRUEGER, P.M.; TRAN, M.K.; HUMMER, R.A.; CHANG, V.W. (2015). Mortality Attributable to Low Levels of Education in the United States. PLoS ONE 10(7): e0131809. DOI: 10.1371/journal.pone.0131809.

LEGER, L. (2001). Schools, health literacy and public health: possibilities and challenges. Health Promotion International 16 (2), 197-205. DOI: 10.1093/heapro/16.2.197.

LINVILLE, HR. (1989). The practical use of biology. School Science and Mathematic, 1909; 9. En Rosenthaal DB. Two Approaches to Science-Tech-
nology-Society (S-T-S). Science Education; 73(5); 581-9. DOI: 10.1111/ j.1949-8594.1909.tb01383.x.

METCALFE, O., ET AL. (1995). La Educación para la salud en el ámbito educativo. Manual de formación para el profesorado y otros agentes educativos. Red Europea de Escuelas Promotoras de Salud. Madrid: Ministerio de Educación y Ciencia y de Sanidad y Consumo.

MGI (McKinsey-Global-Institute) (2014). www.mckinsey.com/insights/. MOLINA, M.C. (2006). Nuevos retos de la educación para la salud: las sociedades multiculturales y las nuevas prioridades. Pedagogía social. Revista interuniversitaria. 12-13, 2, 71-85.

OLIVA, J.M. (2011). Difficulties of secondary school teachers implicating in the reading, innovation and research in science education (I): The problem to begin with. Revista Eureka sobre enseñanza y divulgación de las ciencias, 8(1).

PAAKKARI, L.;TYNJÄLÄ, P.;TORPPA, M.; VILLBERG, J.; KANNAS, L. (2015). The development and alignment of pedagogical conceptions of health education. Teaching and Teacher Education 49, 11-21. DOI: 10.1016/j.tate.2015.02.005.

PEREA QUESADA R. (2001). La educación para la salud, reto de nuestro tiempo. Educación $X X 1,4,15-40$. DOI: $10.5944 /$ educxx1.4.0.361.

RICHONNIER, M. (1990). La contribución de la Comunidad Europea a 
la Educación para la Salud. En T. Williams (Ed.), Actas de la primera conferencia europea de Educación para la Salud y prevención del cáncer en las escuelas (pp. 345-356). Dublín: CEE.

RODRIGO VEGA, M. (1995). Concepciones de los futuros profesores de primaria sobre la Educación para la Salud. Revista Interuniversitaria de Formación del Profesorado, 24, 173-180.

RODRIGO VEGA, M., EJEDA MANZANERA, J.M., CABALLERO ARMENTA, M. (2013). Una década enseñando e investigando en Educación Alimentaria para Maestros. Revista Complutense de Educación. 24, 2, 243-265. DOI: 10.5209/rev_ rced.2013.v24.n2.42078.

SALVADOR LLIVINIA, T. (Coord.) (2008). Diagnóstico de situación sobre avances conseguidos, necesidades y retos en Promoción y Educación para la Salud en la Escuela en España. Madrid: Ed. Ministerio de Sanidad y Consumo.

SANZ GIL, J.J. (2012). Adaptación de las plataformas educativas a la construcción del Espacio Europeo de Educación Superior (EEES). Revista Universitaria Europea, 17, 89-108.

SEFFRIN, J. (2008). Health Education in Schools. The importance of Establishing Healthy Behaviors in Our Nation's Youth. The health Educator Fall, 40 (2) 1-5.

TALAVERA, M.; GAVIDIA, V. (2007). Dificultades para el desarrollo de la educación para la Salud en la escuela. Opiniones del profesorado. Didáctica de las Ciencias Experimentales y Sociales, 21, 119-128.

TALAVERA, M.; GAVIDIA, V. (2013) Percepción de la Educación para la Salud en el personal docente y el sanitario. Didáctica de las Ciencias Experimentales y Sociales, 27, 115129. DOI: $10.7203 /$ dces.27.2569.

THACKERAY, R.;NEIGER, B.L.;BARTLE, H.; HILL, S.C.; BARNES, M.D. (2002). Elementary School Teachers' Perspectives on Health Instruction: Implications for Health Education. American Journal of Health education, 33 (2), 77. DOI: 10.1080/193250 37.2002.10609421.

VEGA FUENTE, A.; ARAMENDI JAUREGUI, P.; BUJÁN VIDALES, M. K.; GARÍN CASALES, S. (2015). La Educación para la Salud en la ESO: Aportaciones de un estudio sobre el País Vasco. Educación $X X 1,18(1), 167-188$. DOI: $\underline{10.5944 /}$ educxx1.18.1.12316.

WHO (1998). Obesity preventing and managing theglobalepidemia. Report of a WHO consultation. Ginebra. DOI: $10.1017 / \mathrm{s} 0021932003245508$.

WYRICK, D.L.; WYRICK, C.H.: BIBEAU, D.L.; FEARNOW-KENNEY, M.D. (2001). Coverage of adolescent substance use prevention in state frameworks for health education. Journal of School Health, 71, 437-442. DOI: 10.1111/j.17461561.2001.tb07320.x. 
YUS, R. (1997). La transversalidad como constructo organizativo del currículo escolar, en la antesala de la globalidad. Investigación en la Escuela, 32, 43-50. 
